Instituto Internacional de Investigación y Desarrollo Tecnológico Educativo INDTEC, C.A.

DOI: https://doi.org/10.29394/scientific.issn.2542-2987.2017.0.0.22.411-429

OAI-PMH: http://www.indteca.com/ojs/index.php/Revista Scientific/oai

\title{
Aproximación Teórica Sustentada en la Autopoiésis para Optimizar la Funcionalidad de la Gerencia en las Escuelas Bolivarianas
}

\author{
Autora: Sonia Elena Garfides González \\ Universidad Nacional Experimental de las Fuerzas Armadas, UNEFA \\ sonigarf2@hotmail.com \\ Yaracuy, Venezuela
}

\section{Resumen}

La presente investigación tuvo como propósito construir una aproximación teórica sustentada en la autopoiésis para optimizar la funcionalidad de la gerencia en las escuelas bolivarianas. Para desarrollar la investigación aborde la realidad mediante un paradigma cualitativo mediante los métodos fenomenológico y hermenéutico. Considere finalmente que los gerentes de las escuelas bolivarianas para lograr la autopoiésis necesitan una aptitud creativa para unirse con su personal la cual amerita el amor, la comunicación efectiva y una praxis concreta comprometidos con una aptitud ética, con la aceptación del otro contra uno con sus virtudes y diferencias en la convivencia incitando la toma de decisiones en consenso mediante la planificación integrada, para lograr la socialización, integración y equilibrio sistémico materializando el pensamiento complejo para imbuir la autopoiésis en el avance y consolidación de los cambios organizacionales en la aplicabilidad de las funciones gerenciales administrativas, pedagógicas y comunitarias en las escuelas bolivarianas.

Palabras clave: autopoiésis; escuelas bolivarianas; gerencia. 


\title{
Sustained Theorical Approach in Autopoiesis to Optimize the Functionality of Management in the Bolivarian Schools
}

\begin{abstract}
The present research aimed to build a theoretical approach based on autopoiesis to optimize the functionality of management in Bolivarian schools. To develop the research, approach reality through a qualitative paradigm through the phenomenological and hermeneutic methods. Finally, consider that the managers of Bolivarian schools to achieve autopoiesis need a creative aptitude to unite with their personnel, which merits love, effective communication and a concrete praxis committed to an ethical aptitude, with the acceptance of the other against one with their Virtues and differences in coexistence by encouraging decision-making in consensus through integrated planning, to achieve socialization, integration and systemic balance materializing complex thinking to imbue autopoiesis in the advance and consolidation of organizational changes in the applicability of functions Administrative, pedagogical and community management in Bolivarian schools.
\end{abstract}

Keywords: autopoiesis; bolivarian schools; management.

Date Received: 11-11-2016

Date Acceptance: 06-01-2017 


\section{Introducción}

El gerente educativo de las Escuelas Bolivarianas se dedica más cumplir con sus funciones administrativas, aislándose del colectivo institucional y comunidad en general evidenciándose ausencia comunicativa y desconocimiento de las acciones pedagógicas y comunitarias que se realizan en la institución que dirige, situación que desmotiva al personal creando un estatismo laboral con situaciones conflictivas por la ausencia del director en la toma de decisiones, a su vez en planificación integrada Proyecto Educativo Integral Comunitario (P.E.I.C.) por delegar sus funciones pedagógicas y comunitarias a los subdirectores y coordinadores, donde el quehacer gerencial juega un papel importante, pues favorece la satisfacción de las exigencias requeridas por su entorno en la búsqueda del bienestar social, a través del logro de la excelencia en su organización.

Es por esa razón, la gerencia en el proceso educativo, debe ir orientada al logro de los objetivos de este nuevo sistema, denominado sistema Educativo Bolivariano y se realiza a través de gerentes educativos dispuestos, lo cual garantiza el compromiso y la responsabilidad de los mismos dentro de las transformaciones sociales y educativas de estos nuevos paradigmas educacionales con ámbitos específicos de generación, tratamiento y diseminación del conocimiento, que implican una gestión de extrema complejidad en un proceso de interacciones recursivas entre ellas y el medio externo, como lo expresa, Maturana (2001), en un influir de cambios estructurales-recíprocos, siendo capaces de conservar su propia organización (autopoiésis), durante su adaptación al medio para afrontar una demanda social de calidad que sostenga el desarrollo actual y futuro, humano, económico y social.

De allí que, en la investigación realizada, pretendió conocer, comprender e interpretar la funcionalidad administrativa, pedagógica y comunitaria de los gerentes en las Escuelas Bolivarianas "María Clementina 
de Bonilla" y "El Salto" del Municipio Peña Estado Yaracuy, como se adaptarían a su entorno para generar la autopoiésis a fin de que manejen cómodamente en armonía situaciones cambiantes de caos y ambigüedades, para promover la innovación mediante un pensamiento complejo creativo. De esta manera se resalta su importancia en los diversos procesos educativos que vive el gerente y las acciones que realiza, poniendo en práctica competencias y destrezas, en vías de mejorar la práctica educativa, así como la gestión pedagógica, comunitaria e institucional.

\section{El Problema}

\subsection{Contextualización y Delimitación de la Investigación.}

Las funciones administrativas, pedagógicas y comunitarias ejercidas por el gerente educativo en Venezuela centrada en una educación de calidad, según Dichter (2001), exige la ruptura de los vínculos autoritarios y unidireccionales. De allí que se requiere de gerentes capacitados en establecer contactos e interacción con su personal, que desarrollen habilidad para mejorar y mantener el esfuerzo en una situación interpersonal educativa, a través de la expresión de la comunicación eficaz que propicie un ambiente favorable para estimular en su personal, la participación, la integración y la convivencia para alcanzar el éxito hacia la calidad educativa.

En este cometido, el gerente educativo necesita contar con suficientes conocimientos gerenciales para lograr una práctica escolar que permita optimizar el ambiente laboral y lo que es más importante, ofrecer una atención adecuada al educando. Al respecto es importante que los gerentes educativos estén al tanto de los diversos cambios y transformaciones educativas, de modo que apropiándose de los nuevos planteamientos y teorías en el ámbito de la gerencia educacional alcance la debida efectividad y mejore en gran medida la capacidad de respuesta a brindar tanto al personal como al contexto escolar en su conjunto. 
De manera que, la gerencia con una visión de pensamiento complejo, expresa Morín (2004), establece "la solidaridad entre las cosas y la fraternidad entre los seres humanos. Estamos en la prehistoria del espíritu humano y solo el pensamiento complejo nos permitirá civilizar nuestro conocimiento" (pág. 28).

En este orden de ideas, considerando el pensamiento complejo en función de fortalecer nuestro conocimiento destaco la conceptualización de sistema que nos ofrece Luhmann (2002), el conocimiento adquiere connotación constructivista y es visto como un acto de creación en el mundo por la observación y la distinción, la observación utiliza la diferencia para designar un lado de aquello que se observa, es por tanto una operación que utiliza una distinción para indicar un lado y no otro. Este es una operación con dos elementos, la distinción y la indicación que no pueden confundirse ni separarse operativamente como plantea García (2004) "en este sentido la observación como método, es el reconocimiento, el reflejo de una estructura subyacente de la comunicación, la comunicación es por tanto creadora de la sociedad." (pág. 4). De allí que la comunicación es un operar social que se entrelaza a otras operaciones de su propio tipo dejando fuera otras cosas, el proceso de autoconstrucción, autoreproducción, autoorganización y autopoiésis.

Desde esta perspectiva, Salas ( 2002), expone que "la mayoría de los gerentes educativos, se dedican solamente a asuntos administrativos y a mantener el orden dejando de lado la esencia del proceso como lo es el proceso pedagógico, así como también el comunitario" (pág. 23) esto permite suponer que para muchos gerentes educativos el mantener el orden en los planteles, es su función primordial, quizás de mayor relevancia y en algunas ocasiones no toman en cuenta la función pedagógica y comunitaria, como por ejemplo, el acompañamiento pedagógico en el aula, visitas a la comunidad en 
diversas circunstancias, con los que garantice una verdadera gestión educativa de calidad.

En relación a lo antes mencionado el motivo que me incentivo a realizar esta investigación, fue la operatividad de los gerentes en las Escuelas Bolivarianas en cuanto a su funcionalidad administrativa, pedagógica y comunitaria, donde no difunden la participación del personal y la comunidad, no formulan ni gestionan la discusión de los elementos del P.E.I.C. (Proyecto Educativo Integral Comunitario) con todo el personal de la comunidad, la usencia del director en los acompañamientos pedagógicos a los docentes para orientar y evaluar el proceso pedagógico, no promueven el trabajo en equipo debido a que delegan sus funciones a los subdirectores y coordinadores $\sin$ interesarse en el procedimiento sino en los resultados para ordenarles sus decisiones que son contradictorias en la mayorías de los casos por falta de conocimientos, ocasionando así niveles bajos de productividad, desmotivación del personal, ausentismo de la comunidad en las escuelas, estatismo, bifurcación, conflictos que desestabilizar la armonía laboral y finalmente desmejora la calidad educativa. Esta situación objeto de estudio de las instituciones educativas reclama cambios educativos autopoiéticos, para regular las diferencias del gerente con el entorno o medio interno y extremo.

Desde esta perspectiva, considere conveniente iniciar mi investigación para comprender e interpretar las funciones administrativas pedagógicas y comunitarias de los gerentes, a fin de generar una aproximación teórica sustentada en la autopoiésis para la funcionalidad de la gerencia en las Escuelas Bolivarianas, en vista de la importancia que tienen dichas escuelas, las cuales fueron creadas según la Resolución № 179, en Septiembre de 1.999, por el Ministerio de Educación y Deportes, uno de los aportes fundamentales es el desarrollo de proyectos dirigidos al contexto social y a la comunidad donde se ubica la escuela. 
En atención a ello, el estudio que se presente estaba orientado a las Escuelas Bolivarianas, donde como investigadora realice conversaciones con directores, docentes y representantes del Municipio Peña Estado Yaracuy permitiéndole realizar un análisis situacional, notándose un contexto contradictorio con la funcionalidad administrativa, pedagógica y comunitaria de los directores para gerenciar ya que se presume poca fluidez en la toma de decisiones, delegación de funciones no compartidas, poca comunicación, dejar pasar las situaciones sin enfrentarlas en el momento que se presentan, planificación estandarizada, dedicación exclusiva a lo administrativo y poca atención hacia los aspectos pedagógicos y comunitarios.

De allí deduzco que el gerente reduce su actuación al cumplimiento de funciones centralizando su acción y actuación a como el piensa y dice; sin considerar la opinión del colectivo en consenso, repercutiendo de esta manera en fallas, malos entendidos, incomodidades entre el personal, provocando así un proceso cíclico con poca comunicación y aislado de las necesidades e intereses del colectivo. Desvalorizando así los planteamientos teóricos de las Escuelas Bolivarianas.

Las consideraciones anteriores me permitieron evidenciar la necesidad de conocer la funcionalidad administrativa, pedagógica y comunitaria de los gerentes en las escuelas Bolivarianas "María Clementilla de Bonilla" y "El Salto", para comprender e interpretar como los gerentes se adaptarían a su entorno para generar la autopoiésis a fin de que manejen cómodamente en armonía situaciones cambiantes y ambigüedades, para promover la innovación mediante un pensamiento complejo creativo. De allí surgen las siguientes interrogantes:

\subsection{Interrogantes iniciales de la investigación}

¿Cuál es el aporte de las teorías relacionadas con la autopoiésis, gerencia y Escuelas Bolivarianas? 
¿Cómo actúan los gerentes de las Escuelas Bolivarianas en su funcionalidad administrativa, pedagógica y comunitaria para generar la autopoiésis?

¿Consideran los gerentes de las Escuelas Bolivarianas el medio externo e interno con conectividad autopoiética?

\section{Objetivos de la Investigación.}

\subsection{Objetivo General.}

Construir una aproximación teórica sustentada en la autopoiésis para la funcionalidad de la Gerencia en las Escuelas Bolivarianas.

\subsection{Objetivos Específicos}

Comprender el aporte de las teorías alusivas a: autopoiésis, gerencia y Escuelas Bolivarianas.

Conocer la funcionalidad administrativa, pedagógica y comunitaria de la gerencia en las Escuelas Bolivarianas.

Interpretar la funcionalidad de la gerencia en las Escuelas Bolivarianas y la conectividad dinámica - autopoiética con el medio externo e interno.

Construir por análisis, interpretación y contraste una aproximación teórica sustentada en la autopoiésis para la funcionalidad de la gerencia en las Escuelas Bolivarianas.

\section{Marco Teórico}

\subsection{Antecedentes Relacionados con la Investigación.}

López (2000), hizo una investigación de Diseño Autopoietico para el desarrollo de las Unidades Generadoras de recursos en la Universidad Centroccidental "Lisandro Alvarado". Universidad Bicentenaria de Aragua. Tesis Doctoral. 
La autora opina en los resultados del estudio que se señalaron las necesidades presentes en la UCLA, las cuales demandan acciones inmediatas, de inmanencialidad del Consejo Universitario, de consubstancialidad del Rectorado y aplicación de las llaves que conducen a la autopoiésis.

Así, la Gnoseología de la autopoiésis a las ciencias biológicas y por analogía a las ciencias sociales, indican que es pertinente la autoproducción en la UCLA y autor reproducción representada en las Unidades Generadoras de Recursos en su identidad como sistemas, la capacidad homeostática, el dominio fenomenológico de interacciones donde las acciones se materializan en la praxis como una alternativa para la autogestión de las Unidades Generadoras de Recursos.

\subsection{Teoría de la Autopoiésis.}

Para Maturana (ob.cit) la propiedad de los sistemas de producirse a sí mismos es la autopoiésis y define el "acoplamiento" de un sistema a su entorno, la autopoiésis es la propiedad básica de los seres vivos puesto que son sistemas determinados en su estructura, es decir, son sistemas tales que cuando algo externo incide sobre ellos, los efectos dependen de ellos mismos, de su estructura en ese instante, y no de lo externo. Los seres vivos son autónomos, en los que su autonomía se da en su autorreferencia y son sistemas cerrados en su dinámica de constitución como sistemas en continua producción de sí mismos.

De acuerdo a Maturana y Varela (2000), un ser vivo es un sistema autopoietico organizado como una red cerrada de producciones moleculares, en la que las moléculas producidas generan la misma red que las produjo, y especifican su extensión. La autopoiésis es la manera de existir de un sistema viviente y su manera de ser una entidad autónoma. Como tal, los sistemas vivientes viven tanto como conserven su organización, y todos sus cambios 
estructurales ocurren con la conservación de su adaptación al medio en el cual ellos existen.

Además, Maturana (ob.cit) piensa que la existencia y la conservación de la auto-identidad humana es un fenómeno social derivado de la existencia humana en el lenguaje. $Y$ también piensa él que es debido que el sí-mismo (self) es una manera de existir en el lenguaje, es posible cambiar al sí-mismo a través del lenguaje, y de ahí que la terapia es posible. Por supuesto que todo esto toma lugar dentro de los límites de la conservación de la autopoiésis, porque si no, el ser vivo se muere. De acuerdo a Maturana (ob.cit), el determinismo estructural, como una abstracción de las coherencias de la experiencia del observador, es anterior a la noción de autopoiésis porque es necesario aceptarla para entender a los sistemas vivientes como sistemas autopoieticos.

De manera que, Maturana (ob.cit) hace referencia al determinismo estructural cuando él menciona a los seres vivos como sistema autopoietico, el habla de sus dos dominios de existencia. Cuando considera los dos dominios de existencia, el habla del espacio de las relaciones y cuando considera este último, y la fisiología, puede hablar de la identidad de un sistema viviente como unos fenómenos sistemáticos, y ya no necesita apelara la autopoiésis.

\subsection{Gerencia}

El termino gerencia es difícil de definir, significa cosas diferentes para personas diferentes. Algunos lo identifican con funciones realizadas por Directores, Empresarios, Gerentes o Supervisores, otros lo refieren a un grupo particular de personas. Para los trabajadores según Sisk y Sverdilik (1979) "gerencia es sinónimo de autoridad sobre el hecho laboral". (pág. 21)

Dado que la dificultad antes mencionada generalmente es omitida, al hablar de gerencia para referirnos a un número indeterminado de acciones y 
funciones que incluyen ser director, administrador, supervisor, planificador, controlador, sin que parezca posible identificar algunos elementos de diferenciación que faciliten la identificación de lo que se denomina la actividad gerencial, la multiplicidad de acepciones es tal que se llega a denominar gerente a quien realiza cualquier actividad de ventas. Para algunos autores una manera de captar la complejidad de la administración es entender que los gerentes pueden trabajar en diferentes niveles de una organización y de diferentes rangos de actividades dentro de ella.

Desde esta perspectiva, la gerencia incorpora los valores intelectuales de las personas que integran la organización entendida como coordinación de operaciones individuales, lo cual implica que considerar al hombre como sistema humano complejo, es reconocerlo como sistema autoorganizado que es capaz de combinar un número muy grande de unidades, pero también de incertidumbres, indeterminaciones, fenómenos aleatorios, es decir según Morin (2001) la aceptación de los sistemas "semialeatorios es inseparable de los azares que la incluyen" (pág. 60).

\section{Metodología de la Presente investigación}

\subsection{Tipo de la Investigación.}

El fundamento de la presente investigación remitió a la adopción de un paradigma cualitativo, a través del cual se pretendió la integración de los métodos fenomenológico y hermenéutico, estos permitieron conocer, comprender e interpretar las observaciones y experiencias detalladas para darles significado a las categorías de análisis a estudiar.

En este sentido, la investigación la realice con la aplicación de los métodos fenomenológico y hermenéutico permitió estudiar fenómenos tal cual como son experimentados y percibidos por el individuo en el contexto donde se estudiará la realidad en la Escuela Bolivariana "El Salto" con características como sistema abierto y la Escuela Bolivariana "María Clementina de Bonilla" 
con características como sistema cerrado, luego se contrastaron ambas realidades a fin de interpretar y comprender las categorías de análisis para teorizar.

Para Sadin (2003), el método fenomenológico "busca la comprensión inmediata del mundo vital del hombre a través de una interpretación global de las situaciones cotidianas" (pág. 32).

En relación al método hermenéutico según Hurtado y Toro (1998), "es el proceso por medio del cual se conoce la vida psíquica con la ayuda de los signos sensibles que son su manifestación, tiene como misión descubrir los significados de las cosas, interpretar las palabras escritas, gestos, pero conservando la singularidad del contexto de que forma parte" (pág. 28).

\subsection{Escenario de investigación e informantes claves.}

Atendiendo al marco cualitativo de la investigación, en cuanto a la selección de informantes claves, escogí dos directoras, dos docentes dos representantes de la comunidad, para un total de seis sujetos de investigación, las cuales poseen información valiosa sobre el tema en estudio y se conocían como representantes de las opiniones y experiencias del colectivo institucional pertenecientes a las Escuelas Bolivarianas "El Salto" y "María Clementina de Bonilla", es relevante destacar que estas instituciones educativas fueron el escenario de investigación.

\subsection{Procedimiento de la investigación.}

El diseño que realice mediante procedimientos a seguir para lograr los objetivos propuestos en la investigación, se contemplan cuatro fases:

Fase I Conceptual inicial. Realice mediante la revisión bibliográfica de los diferentes aspectos investigados por diversos aspectos investigados por diferentes autores, los cuales sustentaron este estudio. En el mismo presento aspectos teóricos importantes para sustentar las categorías de análisis que 
tome en cuenta para la estructuración de la construcción de la aproximación teórica. Así mismo en la primera instancia revise todo el material relacionado con las diferentes teorías que fundamentan el aspecto relacionado con la autopoiésis y gerencia.

Fase II Aplicación de entrevistas. Aplique entrevistas diagnósticas y a profundidad a los informantes claves, con la finalidad de abordar los conocimientos que poseen con respecto a la realidad estudiada, tomando en cuenta el siguiente procedimiento: en las entrevistas realizadas considere los objetivos y las categorías de análisis por una primera instancia, recogí la información, la registre, analicé y redacte informes necesarios para que las personas involucradas puedan revisar y validar. Este procedimiento lo hice de manera cíclica hasta que saturé la información, para el producto final.

Fase III observación participante y diálogos. Además de utilizar la observación participante, use el proceso de diálogo mediante el cual materialice la epojé a fin de aceptar sus propias vivencias con la directora, un docente, un representante de la comunidad, pertenecientes a las Escuelas Bolivarianas "El Salto" y "María Clementina de Bonilla".

Fase IV Construcción teórica final. Finalmente, con las informaciones que obtuve en su totalidad, es decir, que no se dispusieron de nuevos contactos con las fuentes, estos fueron integrados o agrupados en categorías amplias y comprensivas que surgieron de diversos escenarios, introduciendo e interpolando más conceptos teóricos y relaciones complejas entre ellas, con gran énfasis de imaginación creativa. Con el insumo de las entrevistas, se generó la aproximación teórica sustentada en la autopoiesis que permitió comprender e interpretar la aplicación de la funcionalidad de la gerencia en las Escuelas Bolivarianas "El salto" y "María Clementina de Bonilla". 


\subsection{Técnicas e Instrumentos de Recolección de la Información.}

Las técnicas de recolección que utilice fueron las entrevistas diagnósticas y a profundidad y la observación participativa. En este sentido, de acuerdo a los objetivos planteados en la investigación, las técnicas de recolección de información, aplique las entrevistas abiertas y personales en dos fases: (1) entrevista diagnóstica: que realice mediante un lenguaje coloquial de diálogo libre con los sujetos en estudio, asumí una actitud receptiva, para así lograr con el entrevistado un ambiente de confianza, para que pudieran hablar libremente y facilitar que se expresaran en un marco de experiencia vivencial, (2) entrevista en profundidad: la cual consistió en una conversación o encuentro cara a cara, que tuvo como propósito conocer en detalle lo que pensaban o sentían los informantes claves referente al tema en estudio, con la finalidad de descubrir de forma personal los conocimientos que ellos mismos poseían, de la funcionalidad del gerente en las Escuelas Bolivarianas.

No obstante, el tipo de entrevista que utilicé fue semi-estructurada en profundidad, con un guión para asegurar la inclusión de todos los aspectos de interés para la investigación, los cuales no sufrieron modificación de acuerdo a como se desarrolló la entrevista. Con respecto a la observación participativa, usé el diálogo para recabar la información también utilicé grabadora y una libreta para registrar toda la información para completar el registro.

\subsection{Técnicas de análisis y teorización.}

La información aportada por los informantes claves a través de las entrevistas diagnostica y profundidad, fue reducida y categorizada con el uso de la técnica de análisis de contenido de la triangulación por fuente de datos, esto implicó la obtención de relatos acerca de una situación dada desde tres puntos de vista, los relatos de los informantes, los fundamentos teóricos y mi experiencia, estas recopilaciones me ayudaron a realizar un control cruzado 
entre las diferentes fuentes para así obtener y comprobar resultados, de esta manera logré una interpretación más comprensiva sobre el fenómeno de estudio.

Posteriormente con el apoyo de las teorías de insumos y de servicio de la interpretación de la información o hallazgo de la información se generaron reflexiones teóricas acerca de la autopoiésis para la funcionalidad del gerente en las Escuelas Bolivarianas.

\subsection{Análisis e interpretación de resultados.}

La funcionalidad administrativa, pedagógica y comunitaria de los gerentes en las Escuelas Bolivarianas permiten a su personal la dependencia o independencia, la creatividad o el estatismo, la convivencia o la entropía, orden y desorden, las certezas e incertidumbres, lo separable e inseparable. No se trata de abandonar los principios de la ciencia clásica de orden, separabilidad y lógica, sino de integrarlos en un esquema que es, al mismo tiempo más amplio y más rico, no se trata de oponer un holismo global y vacío a un reduccionismo sistemático; se trata de incorporar lo concreto de las partes a la totalidad.

De manera que, el gerente debería articular el orden y el desorden, la separación y la unión, la autonomía y la dependencia, que son al mismo tiempo, complementarios, competidores y antagonistas, en este sentido el pensamiento complejo permite un procedimiento autopoietico que consiste en una ida y vuelta incesante entre certezas e incertidumbres, entre lo elemental y global, entre lo separable e inseparable.

Desde esta perspectiva, en función de la autopoiésis donde las certezas e incertidumbres del sistema a su entorno, se acoplan con la capacidad de los sistemas de reproducirse a sí mismos para no desaparecer, absorbiendo energía de su medio permanentemente. Desde esta perspectiva, considero que es urgente que los directores de las escuelas bolivarianas en estudio, 
operen con un pensamiento complejo mediante la unión del personal docente y representantes interactuando con ellos desde el amor, la armonía y la comunicación efectiva, en virtud de la materialización eficaz y eficiente de sus funciones gerenciales administrativas, pedagógicas y comunitarias, religando así un entramado rico de enredos para unir los conocimientos separados y situarlos en un contexto global.

En este contexto, la ejecución de presente estudio se relacionó directamente con esta realidad, tan compleja e interesante, como es la autopoiesis, seleccionando 6 informantes claves constituidos por 2 directoras, 2 docentes y 2 representantes, para conocer la funcionalidad administrativa, pedagógica y comunitaria de la gerencia en las escuelas bolivarianas.

En relación a los objetivos propuestos, para el análisis, categorización e interpretación de la información recopilada entre los seis informantes claves, se planificaron y se realizaron unas entrevistas en dos fases, fase I diagnóstica la cual se cumplió y se desarrolló en un ambiente jovial con cada informante clave. Cabe destacar que las primeras interpretaciones sirvieron de insumo para las entrevistas sucesivas, las cuales finalmente me remitieron a la teorización que sustentó la aproximación teórica propuesta. Seguidamente, se cumplió la fase II de profundización, en esta se partió de una guía de entrevista que reflejó los conocimientos y experiencias de los informantes claves, respecto al significado que le dan a la funcionalidad de la gerencia de las directoras y se corroboró la información con el aporte de los docentes y representantes de las escuelas bolivarianas "EI Salto" y "María Clementina de Bonilla".

Es relevante destacar, que dicha información se organizó en categorías de análisis que se originaron del entrecruce, de las interrelaciones, las contrastaciones y las triangulaciones entre las informaciones recopiladas entre los informantes claves emergió la aproximación teórica de la presente investigación. 


\section{Reflexiones Conclusivas}

Al evidenciar el caos en el accionar del gerente educativo, es necesario ser creativo e innovador para que desde el amor, la motivación y la tolerancia acepte al otro como diferente adaptándose así al medio interno y externo mediante la materialización inmediata de la autopoiésis con un pensamiento complejo holístico, en función de experimentar ideas creativas y tomar nuevos riesgos en un espacio y tiempo oportuno cuando la situación de caos se presente, ante la incertidumbre de múltiples puntos de vista simultaneas y mundos diferentes, en ese instante el gerente debe estar listo para lo inesperado y deseoso arriesgando su propio punto de vista para consensuar ideas al enfrentar una situación conflictiva.

Atender las situaciones individuales del personal en espacios adecuados para dialogar personalmente, evitando así intimidar e irrespetar al colectivo institucional satisfaciendo las inquietudes desde el amor y comprensión de acuerdo a la situación que se presente.

Integrarse activamente el gerente educativo es neurálgico para involucrarse en los encuentros pedagógicos y planificación integrada (P.E.I.C.) con el colectivo institucional, dejando el miedo o temor a equivocarse partiendo de una gama de acciones que permitan religar, integrar y complementar los aportes de todo el personal en la toma de decisiones, teniendo siempre presente equilibrar con la negentropia o información que sustente los acuerdos consensuados, a fin de propiciar una comunicación efectiva autopoiética en la búsqueda sinérgica de estrategias para optimizar la práctica, pedagógica, admirativa y comunitaria para optimizar sus funciones gerenciales.

\section{Referencias}

Dichter, T. (2001). ¿Es usted un buen gerente? Bogotá: McGraw-Hill. 
Diegoli, S. (2003). El comportamiento de los grupos pequeños de trabajo bajo la perspectiva de la complejidad: Modelos descriptivos y estudio de casos. Universidad de Barcelona. Tesis Doctoral.

García, M. (2004). El conocimiento en construcción. Barcelona: Editorial Gedisa.

Hurtado, L. y Toro j. (2001). Paradigmas y métodos de investigación en tiempos de cambios. Valencia, Venezuela: Episteme.

Luhmann, N. (2002). Complejidad y Modernidad. De la unidad a la diferencia. Editorial Trotta. Madrid, España.

Maturana, H. (2001). El entendimiento y la práctica de la educación. Editorial Universitaria. Santiago de Chile.

Maturana, H. y Varela F. (2002). El árbol de conocimiento. Editorial Universitaria. Santiago de Chile.

Morín. E. (2001). Introducción al pensamiento complejo. Barcelona, España. (5ta Edición. Editorial Gedisa S. A.

Morín E. (2004). Ciencia y Conciencia. Barcelona, España: Editorial Antrophos.

Sandin, M. (2003). La investigación cualitativa en Educación. Fundamentos y tradiciones. Madrid: McGraw-Hill.

Salas (2002). Gerencia Educativa e Innovación. Bogotá, Colombia: Grupo Norma.

Tedesco, E. (2003). Tendencias actuales de la reforma educativa. Boletín número 35 del proyecto de educación UNESCO. Buenos Aires: Gel. 


\section{Sonia Elena Garfides González}

e-mail: sonigarf2@hotmail.com

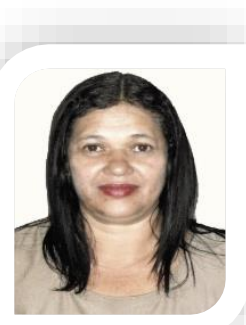

Venezolana, realizó estudios universitarios obteniendo

el título de profesora en educación integral mención: educación para el trabajo egresada de la Universidad Pedagógica Experimental Libertador (U.P.E.L.-I.P.B.). Magister en educación técnica (U.P.E.L.-I.P.B.). Doctora en Ciencias Gerenciales Universidad Nacional Experimental Politécnica de la Fuerza Armada Nacional (U.N.E.F.A.) actualmente cursa Postdoctorado en estudios libres Universidad Fermín Toro (U.F.T.). Docente universitaria, tutora, jurado de tesis de grado en la (U.F.T.), (U.N.E.F.A.), (U.N.E.R.G.).

El contenido de este manuscrito se difunde bajo una Licencia de Creative Commons ReconocimientoNoComercial-Compartirlgual 4.0 Internacional 